\title{
Estudo dos conhecimentos de pacientes com hipertensão, diabetes ou glaucoma sobre suas doenças
}

\author{
Levelofknowledge of patients with glaucoma about their diagnosiscompared with that \\ of hypertensive and diabetic patients
}

\author{
Kenji Sakata ${ }^{1}$ \\ Silvane Bigolin² \\ Agostinho Bryk Junior ${ }^{2}$ \\ Maria Claudia Gomes Komatsu² \\ Lisandro Sakata ${ }^{2}$ \\ Leciana Rorato Chiconelli Vanzo ${ }^{3}$ \\ Hilton Iran Ruthes ${ }^{3}$
}

\section{RESUMO}

Objetivo: Investigar o nível de conhecimento dos pacientes com diagnóstico de glaucoma sobre a sua doença, bem como comparar com o número de pacientes com suspeita de hipertensão ou diabetes com conhecimento da sua doença. Métodos: Realizou-se estudo descritivo durante a primeira etapa do Projeto Glaucoma no ano de 1999 no município de Piraquara-PR, onde foi atendida a população local acima de 40 anos realizando-se exame oftalmológico (tonometria, fundoscopia), aferição da pressão arterial e teste de glicemia. Resultados: Foram atendidas 922 pessoas. Duzentos e seis pacientes foram triados como suspeitos de serem diabéticos, dos quais $42,72 \%(n=88)$ sabiam de sua doença. Encontraram-se 625 pacientes com suspeita de hipertensão, dos quais $60,64 \%(n=379)$ afirmavam serem hipertensos. Foram encontrados 150 pacientes com suspeita de glaucoma crônico simples sendo que destes, apenas 3,33\% $(\mathrm{n}=5)$ sabiam de sua doença, ao passo que $96,67 \%(n=145)$ a desconheciam. Conclusão: O nível de conhecimento de pacientes com diagnóstico de glaucoma é extremamente baixo comparado com o número de pacientes com suspeita de terem hipertensão arterial ou diabetes.

Descritores: Hipertensão/patologia; Diabetes mellitus/patologia; Glaucoma/patologia Estudos de avaliação; Adulto

\section{INTRODUÇÃO}

O glaucoma é uma doença crônica, insidiosa, que pode levar a cegueira ${ }^{(1)}$.

As causas mais freqüentes de cegueira incluem catarata, degeneração macular senil, glaucoma e retinopatia diabética. Os custos para a prevenção da cegueira induzida por estas condições são significativamente menores que os custos gastos na manutenção de um cego na idade adulta ${ }^{(2)}$.

Para a prevenção do glaucoma, uma patologia prevenível mas não recuperável, é imprescindível que os pacientes conheçam a doença e disponham de atendimento adequado para o diagnóstico precoce, trazendo a população para perto do oftalmologista.

O objetivo do presente estudo foi investigar o nível de conhecimento de pacientes com diagnóstico de glaucoma sobre a sua doença, bem como comparar com o número de pacientes com suspeita de hipertensão ou diabetes que tinham conhecimento da sua patologia.

\section{MÉTODO}

Realizou-se um estudo descritivo durante a primeira etapa do Projeto 
Glaucoma do Hospital de Clínicas da Universidade Federal do Paraná, no ano de 1999 no município de Piraquara - PR, cidade da região metropolitana de Curitiba. Segundo censo do IBGE de 1996, o município possui uma população de 56.057 habitantes, com 10.485 habitantes com mais de quarenta anos. Este projeto visa a divulgação de informações sobre o glaucoma, o atendimento oftalmológico, além de realizar a aferição da pressão arterial e o teste glicêmico da população local com idade superior a 40 anos. Participam do projeto: oftalmologistas, residentes em oftalmologia e acadêmicos de medicina.

Todos os pacientes atendidos na unidade de saúde do município foram submetidos à anamnese com ênfase em queixas oftalmológicas e na história pregressa de hipertensão, diabetes e glaucoma. Realizou-se a dosagem da glicemia utilizando tiras reagentes tipo Dextrostix ${ }^{\circledR}$, efetuando-se a leitura no aparelho glucometer. A aferição da pressão arterial foi efetuada com o paciente sentado, após 10 minutos de repouso. Quando os níveis pressóricos apresentavam-se iguais ou superiores a 140x90 mmHg, repetia-se a aferição da PA após 5 minutos.

Avaliou-se a profundidade da câmara anterior através de iluminação oblíqua. Seguiu-se a instilação de uma gota de colírio anestésico e de fluoresceína. Nos pacientes com câmara anterior profunda, administrou-se também uma gota de fenilefrina. A aferição da pressão intra-ocular (Po) se fez com tonômetro de aplanação Haag Streit acoplado à lâmpada de fenda. Realizaram-se duas medidas consecutivas e considerou-se a média entre elas. Seguiu-se a fundoscopia com oftalmoscópio direto e, em casos de suspeita de retinopatia, também a oftalmoscopia indireta binocular. Utilizaram-se como critérios de fundo de olho compatível com glaucoma a escavação maior que 0,5 , a escavação vertical maior que a horizontal, a assimetria de escavação maior que 0,2 , a presença de hemorragias na papila e a presença de "notch". Os pacientes suspeitos de glaucoma de pressão normal ou hipertensão ocular foram chamados para posterior avaliação no Hospital de Clínicas. Nesta ocasião repetiu-se a tonometria e a fundoscopia, e realizou-se também exame da acuidade visual, refração, gonioscopia e paquimetria, onde se selecionaram os pacientes com Po maior que $22 \mathrm{mmHg}$ ou quadro fundoscópico compatível com glaucoma de pressão normal para a realização de campo visual com perímetro computadorizado TOPCON modelo SBP 2020, estratégia 24/2. Adotaram-se os seguintes critérios para o diagnóstico de glaucoma na perimetria: escotoma de Seidel, depressão nasal superior ou inferior, degrau nasal, escotomas arqueados superiores ou inferiores completos ou incompletos.

Os pacientes com alterações na pressão arterial tendo pressão sistólica igual ou acima de $140 \mathrm{mmHg}$, diastólica maior ou igual que $90 \mathrm{mmHg}$ e/ou glicemia superior a $126 \mathrm{mg} / \mathrm{dL} \mathrm{em}$ jejum e $140 \mathrm{mg} / \mathrm{dL}$ pós-prandial foram encaminhados à unidade de saúde ou ao serviço médico que acompanham.

\section{RESULTADOS}

Na primeira etapa do projeto foram atendidas 922 pessoas.

Duzentos e seis pacientes foram triados como suspeitos de serem diabéticos, dos quais $42,72 \%(\mathrm{n}=88)$ sabiam da sua doença e $57,28 \%(\mathrm{n}=118)$ desconheciam a hipótese diagnóstica.

No que tange à pressão arterial, encontraram-se 625 pacientes com suspeita de hipertensão, dos quais $60,64 \%$ $(n=379)$ afirmavam serem hipertensos e 39,36\% $(n=246)$ negavam a patologia.

Em relação ao exame oftalmológico, 150 pacientes apresentaram suspeita de glaucoma de pressão normal ou hipertensão ocular. Esses pacientes foram chamados para posterior avaliação no Hospital de Clínicas, contudo apenas 69 compareceram. Nesta ocasião, realizou-se exame oftalmológico completo, onde se selecionaram 37 pacientes para a realização da perimetria computadorizada. Destes, 36 compareceram ao exame. Vinte e seis pacientes apresentaram alteração do campo visual compatível com o glaucoma (pelo menos em um olho) sendo 11 de glaucoma crônico simples (Po acima de $22 \mathrm{mmHg}$ ) e 15 de glaucoma de pressão normal.

Dos 32 pacientes considerados suspeitos de glaucoma na triagem em Piraquara por apresentarem escavação de $0,6 \mathrm{ou}$ escavação vertical maior que horizontal foram reavaliados na consulta de retorno no Hospital de Clínicas e diagnosticados como normais. Todos eles apresentavam rimas neurais preservadas.

Mesmo sem realizar o exame de campo visual (não compareceram ao atendimento de retorno no HC), oito pacientes foram diagnosticados como portadores de glaucoma segundo os seguintes critérios: Po acima de $28 \mathrm{mmHg}$ (seis pacientes) e escavação maior de 0,8 com "notch" e Po normal (dois pacientes).

Neste estudo, cinco pacientes relataram apresentar glaucoma. Destes, dois pacientes tiveram seu diagnóstico confirmado sendo um deles com fundo de olho e campo visual alterados e o outro com fundo de olho compatível e uso de dois colírios antiglaucomatosos. Em relação aos outros três pacientes, um faleceu três semanas após o primeiro atendimento e outro não pode ser localizado devido à mudança de endereço, sendo ambos com Po normal sem uso de medicação e fundo de olho não característico de glaucoma. O último, apesar de estar em uso de medicação antiglaucomatosa, apresentava fundo de olho normal com escavação de 0,2 e rima neural preservada, sendo a Po de $14 \mathrm{mmHg}$ em ambos os olhos.

Encontramos 18 pacientes com diagnóstico de glaucoma crônico simples de pressão alta e 17 pacientes com glaucoma de pressão normal, totalizando 25 pacientes confirmados.

\section{DISCUSSÃO}

Segundo a Organização Mundial de Saúde (OMS), cerca de $80 \%$ da cegueira presente nos países em desenvolvimento pode ser prevenida ou curada $^{(2-3)}$. O glaucoma representa a 
segunda causa mais comum de cegueira registrada nos Estados Unidos ${ }^{(3-4)}$. Sendo uma causa importante de cegueira potencialmente prevenível, o diagnóstico precoce e a informação da população sobre o glaucoma deveriam fazem parte de programas de saúde comunitária.

Dentro da problemática glaucoma - cegueira um aspecto chama atenção: a falta de conhecimento da população sobre esta patologia, inclusive entre pacientes já em tratamento ${ }^{(5)}$. Nesse estudo, dos 625 pacientes com suspeita de hipertensão arterial, $60,64 \%$ afirmavam serem hipertensos; das 206 pessoas suspeitas de serem diabéticas, $42,72 \%$ conheciam o seu diagnóstico, enquanto dos 150 suspeitos de glaucoma apenas $3,33 \%(\mathrm{n}=5)$ sabiam de sua patologia e dos 35 pacientes confirmados com glaucoma, 5,71\% $(\mathrm{n}=2)$ conheciam seu diagnóstico, mostrando um nível de conhecimento extremamente baixo dos pacientes com glaucoma sobre seu diagnóstico. É importante salientar que 69 pacientes suspeitos ainda não realizaram o campo visual.

Em um estudo observou-se que mesmo entre pacientes em tratamento e acompanhamento em um ambulatório de glaucoma, apenas $30 \%$ têm consciência de serem portadores de glaucoma $^{(5)}$.

Em outro estudo verificou-se que $42 \%$ dos pacientes negros e $50 \%$ dos pacientes brancos com glaucoma de um programa de triagem conheciam seu diagnóstico e encontrava-se em tratamento ${ }^{(6)}$. Estes números contrastam com os resultados encontrados em nosso estudo, onde apenas 3,33\% dos pacientes com suspeita de glaucoma e $5,71 \%$ dos confirmados conheciam seu diagnóstico, mostrando que em nosso meio o glaucoma ainda é uma doença pouco popular. Comparando-se com outras patologias crônicas e que atingem principalmente a população acima de 40 anos como a hipertensão e o diabetes, o glaucoma representa uma patologia extremamente subdiagnosticada, provavelmente devido às dificuldades de acesso da população ao médico oftalmologista e também pela dificuldade dos oftalmologistas em detectar lesões glaucomatosas precoces no exame de fundo de olho. Como preconiza Remo Suzanna Jr, o médico oftalmologista deve estar apto a realizar um exame fundoscópico minucioso a fim de que lesões precoces de glaucoma, principalmente de glaucoma de pressão normal, não passem desapercebidas ${ }^{(7)}$.

Concluiu-se, apesar de nossa pequena amostra, que o nível de conhecimento de pacientes com diagnóstico de glaucoma é extremamente baixo comparado com o número de pacientes com suspeita de terem hipertensão ou diabete e principalmente comparado com o conhecimento de pacientes de países desenvolvidos ${ }^{(8-13)}$.

\section{ABSTRACT}

Purpose: To investigate the level of knowledge among patients with glaucoma about their disease and to compare this group with the number of patients with suspected hypertension or diabetes who know about their disease. Methods: A descriptive study was conducted during the first stage of the Glaucoma Project in 1999 in the municipality of Piraquara, PR, where the local population older than 40 years was submitted to ophthalmologic examination (tonometry and fundoscopy), measurement of arterial pressure, and blood glucose test. Results: A total of 922 individuals were examined. Two hundred and six patients were screened as suspected diabetics; of these, $42.72 \%(\mathrm{n}=88)$ knew about their disease. We detected 625 patients with suspected hypertension, $60.64 \%$ of whom $(\mathrm{n}=379)$ declared that they were hypertensive. A hundred and fifty pacients were screened as suspected open angle glaucoma; of these, only $3.33 \%(n=5)$ knew about their disease and $96.67 \%(n=145)$ were not aware of their disease. Conclusion: The level of knowledge of patients with a diagnosis of glaucoma is extremely low compared to the number of patients with suspected arterial hypertension or diabetes.

Keywords: Hypertension/pathology; Diabetes mellitus/pathology; Glaucoma/pathology; Evaluation studies; Adult

\section{REFERÊNCIAS}

1. Yasuoka ER, Mello PAA, Norris EJ. Quem segue corretamente o tratamento clínico do glaucoma? Arq Bras Oftalmol 1996;59:199-204.

2. Costa VP, Almeida GV, Kara-José N. Prevenção da cegueira por glaucoma. Arq Bras Oftalmol 1998;61:356-60.

3. Gullo RM, Costa VP, Bernardi L, Kara-José N. Condições visuais de pacientes glaucomatosos em um hospital universitário. Arq Bras Oftalmol 1996; 59:147-50.

4. Klein BE, Klein R, Sponsel WE, Frank T. Prevalence of glaucoma. The Beaver Dam Eye Study. Ophthalmology 1992;99:1499-504.

5. Costa VP, Vasconcelos JPC, Pelegrino M, et al. O que os pacientes sabem sobre o glaucoma? Arq Bras Oftalmol 1995;58:36-41.

6. Sommer A, Tielsch JM, Katz J, Quigley HÁ, Gottsch JD, Javitt J, et al. Relationship between intraocular pressure and primary open angle glaucoma among white and black americans - The Baltimore Eye Survey. Arch Ophthalmol 1991;109:1090-5.

7. Susanna Jr R. Nervo óptico no glaucoma: aspectos oftalmoscópicos. São Paulo: Edusp; 1996.

8. Zimmerman TJ, Zalta AH. Facilitating patient compliance in glaucoma therapy. Surv Ophthalmol 1983;28 Suppl:252-8.

9. Mitchell P, Smith W, Attebo K, Healey PR. Prevalence of open-angle glaucoma in Australia. The Blue Mountains Eye Study. Ophthalmology 1996; 103:1661-9.

10. Coffey M, Reidy A, Wormald R, Xian WX, Wright L, Courtney P. Prevalence of glaucoma in the west of Ireland. Br J Ophthalmol 1993;77:17-21.

11. Tielsch JM, Katz J, Quigley HA, Javitt JC, Sommer A. Diabetes, intraocular pressure, and primary open-angle glaucoma in the Baltimore Eye Survey Ophthalmology 1995;102:48-53.

12. Dielemans I, de Jong PT, Stolk R, Vingerling JR, Grobbee DE, Hofman A. Primary open-angle glaucoma, intraocular pressure, and diabetes mellitus in the general elderly population. The Rotterdam Study. Ophthalmology 1996; 103:1271-5.

13. Dielemans I, Vingerling JR, Algra D, MSc, Hofman A, Grobbee DE, de Jong PT. Primary open-angle glaucoma, intraocular pressure, and systemic blood pressure in the general elderly population. The Rotterdam Study. Ophthalmology 1995;102:54-60. 